\title{
CRI iAtlas: an interactive portal for immuno-oncology
}

\section{research [version 1; peer review: 3 approved]}

\author{
James A. Eddy (iD1, Vésteinn Thorsson², Andrew E. Lamb1, David L. Gibbs², \\ Carolina Heimann², Jia Xin Yu³, Verena Chung (D1, Yooree Chae1, Kristen Dang1, \\ Benjamin G. Vincent ${ }^{4}$, Ilya Shmulevich², Justin Guinney ${ }^{1}$
}

\author{
${ }^{1}$ Sage Bionetworks, Seattle, WA, 98101 \\ ${ }^{2}$ Institute for Systems Biology, Seattle, WA, 98109 \\ ${ }^{3}$ Anna-Maria Kellen Clinical Accelerator, Cancer Research Institute, New York, NY, 10006 \\ ${ }^{4}$ University of North Carolina, Chapel Hill, NC, 27599
}

V1 First published: 24 Aug 2020, 9:1028

https://doi.org/10.12688/f1000research.25141.1

Latest published: 24 Aug 2020, 9:1028

https://doi.org/10.12688/f1000research.25141.1

\begin{abstract}
The Cancer Research Institute (CRI) iAtlas is an interactive web platform for data exploration and discovery in the context of tumors and their interactions with the immune microenvironment. iAtlas allows researchers to study immune response characterizations and patterns for individual tumor types, tumor subtypes, and immune subtypes. iAtlas supports computation and visualization of correlations and statistics among features related to the tumor microenvironment, cell composition, immune expression signatures, tumor mutation burden, cancer driver mutations, adaptive cell clonality, patient survival, expression of key immunomodulators, and tumor infiltrating lymphocyte (TIL) spatial maps. iAtlas was launched to accompany the release of the TCGA PanCancer Atlas and has since been expanded to include new capabilities such as (1) user-defined loading of sample cohorts, (2) a tool for classifying expression data into immune subtypes, and (3) integration of TIL mapping from digital pathology images. We expect that the CRI iAtlas will accelerate discovery and improve patient outcomes by providing researchers access to standardized immunogenomics data to better understand the tumor immune microenvironment and its impact on patient responses to immunotherapy.
\end{abstract}

Keywords

genomics, cancer, immunology, systems biology, R, Shiny

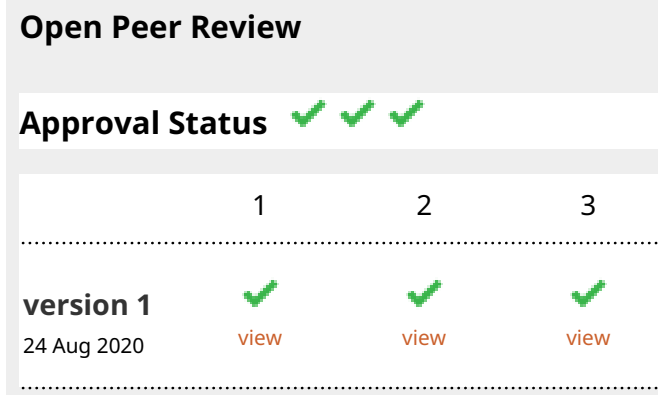

1. Vincent Rouilly, DATACTIX, Bordeaux, France

2. David L. Goode, Peter MacCallum Cancer Centre, Melbourne, Australia University of Melbourne, Melbourne, Australia

3. Nathan E. Reticker-Flynn ID, Stanford University School of Medicine, Stanford, USA Any reports and responses or comments on the article can be found at the end of the article.

This article is included in the Oncology gateway. 
This article is included in the RPackage

gateway.

Corresponding authors: James A. Eddy (james.eddy@sagebionetworks.org), Ilya Shmulevich (ilya.shmulevich@isbscience.org), Justin Guinney (justin.guinney@sagebionetworks.org)

Author roles: Eddy JA: Conceptualization, Data Curation, Funding Acquisition, Methodology, Project Administration, Software, Supervision, Visualization, Writing - Original Draft Preparation, Writing - Review \& Editing; Thorsson V: Conceptualization, Data Curation, Funding Acquisition, Methodology, Project Administration, Software, Supervision, Visualization, Writing - Original Draft Preparation, Writing - Review \& Editing; Lamb AE: Data Curation, Software, Writing - Original Draft Preparation, Writing - Review \& Editing; Gibbs DL: Data Curation, Formal Analysis, Methodology, Software, Writing - Original Draft Preparation, Writing - Review \& Editing; Heimann C: Data Curation, Formal Analysis, Software, Writing - Original Draft Preparation, Writing - Review \& Editing; Yu JX: Data Curation, Formal Analysis, Software; Chung V: Formal Analysis, Software; Chae Y: Project Administration, Writing - Review \& Editing; Dang K: Project Administration, Supervision, Writing - Review \& Editing; Vincent BG: Formal Analysis, Methodology, Supervision, Writing - Original Draft Preparation, Writing - Review \& Editing; Shmulevich I: Conceptualization, Funding Acquisition, Supervision, Writing - Original Draft Preparation, Writing - Review \& Editing; Guinney J: Conceptualization, Funding Acquisition, Supervision, Writing - Original Draft Preparation, Writing - Review \& Editing

Competing interests: No competing interests were disclosed.

Grant information: Funding for this work from the Cancer Research Institute is gratefully acknowledged. Dr. Vincent acknowledges support from the UNC University Cancer Research Fund and a Career Catalyst Research grant from the Susan G. Komen Foundation. The funders had no role in study design, data collection and analysis, decision to publish, or preparation of the manuscript.

Copyright: ( $) 2020$ Eddy JA et al. This is an open access article distributed under the terms of the Creative Commons Attribution License, which permits unrestricted use, distribution, and reproduction in any medium, provided the original work is properly cited.

How to cite this article: Eddy JA, Thorsson V, Lamb AE et al. CRI iAtlas: an interactive portal for immuno-oncology research [version 1; peer review: 3 approved] F1000Research 2020, 9:1028 https://doi.org/10.12688/f1000research.25141.1

First published: 24 Aug 2020, 9:1028 https://doi.org/10.12688/f1000research.25141.1 


\section{Introduction}

Immuno-oncology (IO) is one of the most promising areas of cancer research, with IO-based treatments demonstrating high efficacy within certain cancer types and subsets of patients ${ }^{1-4}$. To broaden the utility of these therapies to more patients, fundamental research is required to improve our understanding of tumor-immune interactions - allowing the next-generation of therapeutics and treatment strategies to emerge $e^{4}$. Advances in the IO field are impeded by the inaccessibility of IO study data and results and lack of data standardization, limiting the ability to easily compare results across studies. This has led to the underutilization of existing data, unnecessary study duplication, and failure to achieve rapid consensus in the field ${ }^{5}$. With the vast increase in the number and scope of IO projects expected in the coming years combined with widespread adoption of genomics and other high dimensional technologies, these problems will be compounded going forward.

We developed the Cancer Research Institute (CRI) iAtlas portal (https://www.cri-iatlas.org) to integrate IO research data, with the goal of providing an interactive, exploratory hub for the IO research community. In doing so, we hope to improve the accessibility and utility of critical resources generated from IO studies. iAtlas is a set of analytic modules-hosted on the web-for studying interactions between tumors and the immune microenvironment. These modules allow researchers to explore associations among a variety of immune characterizations as well as with genomic and clinical phenotypes.

The initial release of iAtlas (April 5, 2018) provided a rich resource to complement analysis results from The Cancer Genome Atlas (TCGA) Research Network on the TCGA data set comprising over 10,000 tumor samples and 33 tumor types ("The Immune Landscape of Cancer"; here referred to as "Immune Landscape"). This study identified six immune subtypes that span cancer tissue types and molecular subtypes, and found that these subtypes differ by somatic aberrations, microenvironment, and survival. Per-sample characterizations included total lymphocytic infiltrate (from DNA methylation as well as H\&E imaging data), estimated cell type fractions, immune gene signature expression, MHC/HLA type and expression, antigen presentation machinery, $\mathrm{T}$ cell and $\mathrm{B}$ cell receptor repertoire inference, viral/microbial characterization, associations with pathway disruption and activity, and other analysis results. The Immune Landscape ${ }^{6}$ manuscript reported on the most novel and potentially therapeutically salient statistical associations between these immune subtypes and the results of the immune characterization. We have continued to develop and evolve the CRI iAtlas application; here, we report the technical design and implementation of iAtlas up to and including the recently released version $1.2^{7}$. This version includes new features requested by users including: (1) user-defined loading of sample cohorts, (2) a tool for classifying expression data into immune subtypes, and (3) integration of TIL mapping from digital pathology images.

\section{Methods}

Implementation

iAtlas is a web-based application to enable data exploration for clinicians, biologists, and informaticists. The inputs and architecture of the application are described below.

Data

The iAtlas app uses structured data and outputs from the Immune Landscape $^{6}$ study and the TCGA PanCancer Atlas initiative ${ }^{8}$, which harmonized TCGA data, ensuring uniform quality control and sample inclusion, batch effect detection, normalization across platforms, combination mutation calling from multiple centers, and robustly compiled clinical and outcome data. A key source of iAtlas data is the table summarizing tumor-sample and immune characterizations for 11,080 TCGA patient participants of the TCGA, Table S1 of the Immune Landscape ${ }^{6}$ manuscript, here termed the "PanImmune Feature Matrix". Auxiliary data were sourced from files available on this manuscript's data page at the NCI Genomic Data Commons, from the TCGA PanCancer Atlas Data Mirror, and from the TCGA PanCancer Atlas working space in Synapse (see Data availability). iAtlas data were formatted as data frames (tables) and stored as "feather" files (https:// github.com/wesm/feather) on the application server for fast loading (Table 1).

Table 1. iAtlas data files.

\begin{tabular}{|l|l|}
\hline $\begin{array}{l}\text { Filename } \\
\text { fmx_df.feather }\end{array}$ & Description \\
\hline feature_df.feather & All immune readout features/variables (11,080) across samples (139). \\
\hline $\begin{array}{l}\text { feature_method_df.feather } \\
\text { im_direct_relationships. } \\
\text { feather }\end{array}$ & Additional annotations for a subset (104) features. \\
\hline $\begin{array}{l}\text { im_expr_df.feather } \\
\text { im_potential_factors. } \\
\text { feather }\end{array}$ & Annotation and source of immunomodulators genes (79). \\
\hline $\begin{array}{l}\text { io_target_annotations. } \\
\text { feather }\end{array}$ & Additional genes (97) that have potential to be involved in immune modulation. \\
\hline $\begin{array}{l}\text { io_target_expr_df.feather } \\
\text { sample_group_df.feather }\end{array}$ & $\begin{array}{l}\text { Annotation of immuno-oncology target genes (405). } \\
\text { Immunession for immuno-oncology target genes (401) across samples (9,693) }\end{array}$ \\
\hline
\end{tabular}


Annotation and browsing of the PanImmune Feature Matrix: iAtlas includes a Data Description page with details on all variables presented in individual modules, with the ability for users to "drill down" on related groups of variables to understand how values were derived. Variables are listed in a text-searchable table containing the name of the variable, the 'Variable Class', the unit (if applicable), and whether the variable is numeric or categorical. A 'Variable Class' is the name of a group of variables that are of similar type and are often the result of one particular analysis. Clicking on a row exposes a list of all variables in the 'Variable Class' and provides links to text descriptions of the analysis methods used to generate the variables.

\section{R/Shiny architecture}

iAtlas is powered by Shiny ${ }^{9}$ and makes extensive use of Shiny Modules $^{10}$ to organize code into composable units (Figure 1). Each iAtlas Analysis module is designed as a Shiny module, allowing simple integration of new analytical functionality. iAtlas uses the tidyverse ${ }^{11}$ family of $\mathrm{R}$ packages (e.g., dplyr ${ }^{12}$, tidyr $^{13}$, purrr $^{14}$, stringr ${ }^{15}$, tibble ${ }^{16}$ ) as well as the wrapr ${ }^{17}$ package to assist with tidy evaluation. These functions power the data transformations of internal tabular data that are then used to create the interactive plots (i.e., with the plotly ${ }^{18}$ graphing library) and data tables (via the $D T^{19}$ wrapper to the DataTables library) seen through the iAtlas modules. We also make heavy use of the crosstalk ${ }^{20}$ package to enable event-driven updates

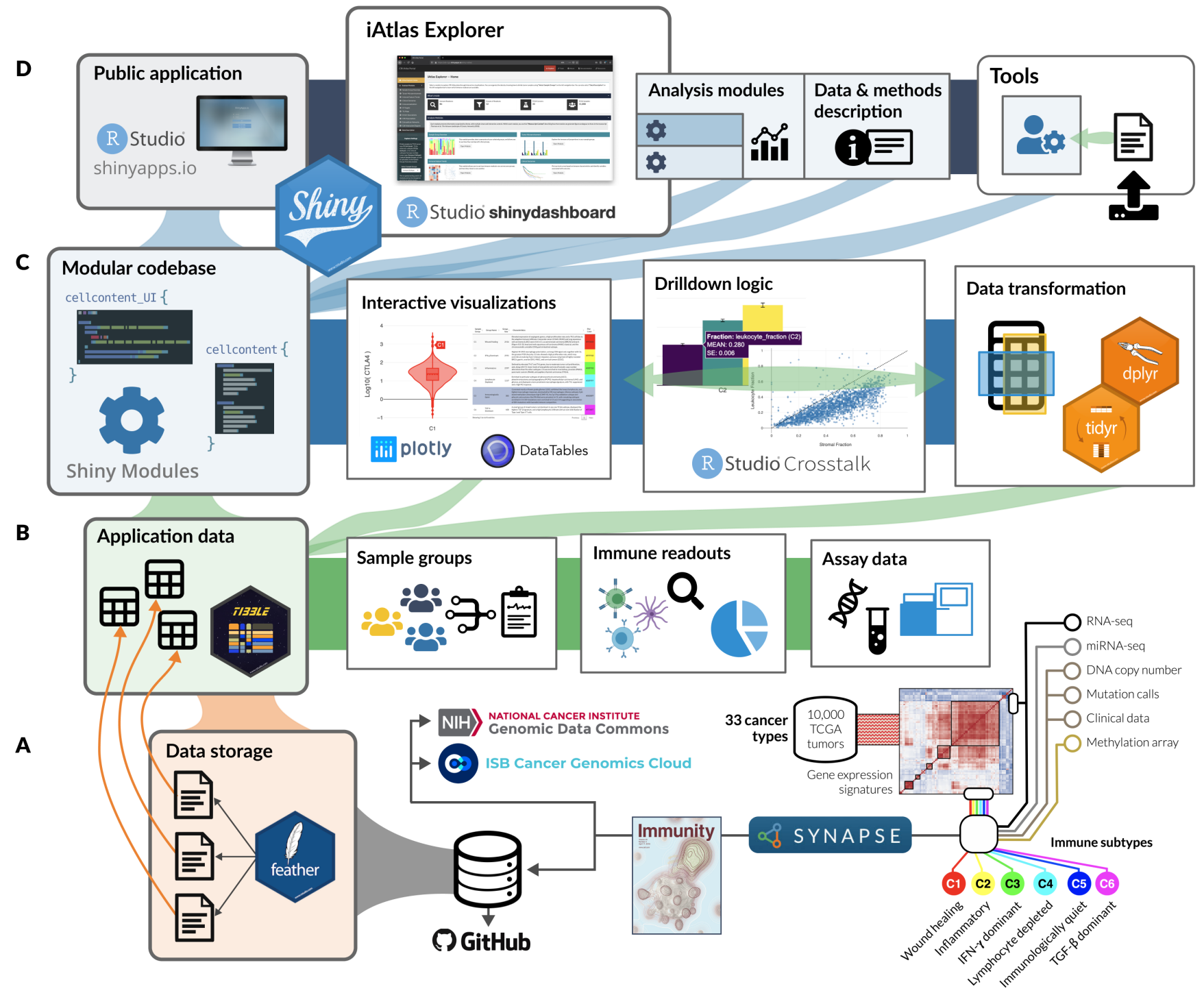

Figure 1. iAtlas architecture overview. (A) Structured data from immunogenomic analyses, including the Immune Landscape ${ }^{6}$ study and expanding over time, are organized and stored as flat (i.e., feather) files within Synapse and made available alongside the application code in GitHub. (B) Tabular data from feather files are read from disk into memory to drive all operations related to sample groupings, sample-level immune characterizations (readouts), and more granular—and high dimensional-assay measurements. (C) The core application code is built as a catalog of Shiny Modules, each of which encapsulates logic for data transformation and visualization related to a scientific theme or assay type. (D) Analysis modules, tools, and data description views are hosted in a unified application on shinyapps.io; the layout and connectivity between modules in the iAtlas Explorer space are managed by the shinydashboard ${ }^{11}$ library. 
to the application state. The core iAtlas application is hosted on https://shinyapps.io.

\section{Analysis modules}

The main feature of the iAtlas interface is the iAtlas Explorer (Figure 2, found under the EXPLORE tab), which provides several Analysis modules to explore and visualize results. Each module supports a type of exploration, with interactive views and controls to enhance and extend the results and analytics as initially described in the Immune Landscape ${ }^{6}$ study. The layout of pages and sections within the iAtlas Explorer is driven by the shinydashboard ${ }^{21}$ package.

Within each module in iAtlas, results are displayed relative to Sample Groups, corresponding to defined study cohorts. Several Sample Groups options are pre-loaded in the tool: first, TCGA tumor type (TCGA Study), which are the standard TCGA tumor types collected and designated by the TCGA. Second, TCGA tumor subtypes (TCGA Subtype), a compendium of further subdivision of TCGA studies into molecular subtypes according to publications by the TCGA Research Network $^{22}$. Finally, a division of tumor samples into distinct patterns of immune response in cancer (Immune Subtypes) is provided $^{6}$. The choice of Sample Groups is global across all modules but can be updated at any time via the Select Sample Groups element in the side menu. We also allow users to upload custom-grouped samples and analyze those with iAtlas modules. The selection of a sample group defines the samples utilized in all analysis modules. For convenience, group annotations can be displayed in visualizations within each module.

Sample Group Overview: View summary information for user-selected sample cohort groups. There are currently three sections: Custom Groups, Group Key, and Group Overlap. Respectively, these sections permit loading of user-defined sample groups, review of detailed annotations of sample groups in a table, and display of overlap between different types of groupings in a mosaic plot.

Tumor Microenvironment: Explore immune cell proportions in sample groups with two sets of faceted bar charts, one for overall cellular proportions (i.e., leukocyte, stromal, and tumor fraction) and one for computed immune cell proportions (e.g., monocytes, CD8+ T cells, naive B cells).

Immune Feature Trends: Visualize how immune readouts vary across sample groups. Violin or box plots show the distribution of individual values across samples in each group, while heatmaps and scatter plots can be used to explore the correlation between any pair of variables within each group.

Clinical Outcomes: Quantify the relationship between immune response and disease outcome, in terms of either overall survival (OS) or progression free interval (PFI) ${ }^{23}$. Results are displayed as Kaplan Meier plots as well as heat maps showing the concordance index between variables and survival.

Immunomodulators: Explore the expression of genes coding for immunomodulating proteins ${ }^{6}$, which include therapeutically important immune checkpoint proteins. Immunomodulators are organized by grouping into three categories: Gene Family (such as "TNF", "MHC Class II", "Immunoglobulin", or "CXC chemokine”), Super Category (such as "Ligand", "Receptor", or "Antigen presentation"), and Immune Checkpoint (classified as "Inhibitory" or "Stimulatory"). Violin and box plots are again used to present distributions, and a table provides additional metadata about immunomodulator genes.

Driver Associations: Test and visualize associations between mutations and IO-related response variables. In the Immune Landscape ${ }^{6}$ study, we reported somatic driver alterations that are correlated with increases or decreases in overall immune cell content, or with the fraction of individual immune cell types. These and other variables can be selected to calculate the significance of relationships in each sample group and view results in a volcano plot.

TIL Maps: We used the results of a recently reported method to assess which spatial regions of hematoxylin and eosin (H\&E) whole slide images show evidence of tumorinfiltrating lymphocytes (TILs) ${ }^{24}$. The method, which uses deep learning, was applied to thousands of H\&E slides of the TCGA, allowing slides to be characterized in terms of TIL density and patterns.

Integration with Landscape of IO Drug Target Development: CRI has compiled and published comprehensive overviews describing ongoing immunotherapy drug trials, including targets, agents, and tumor sites and has made summaries available in an online resource, the Immune-Oncology Landscape (IO Landscape) (www.cancerresearch.org/IO-landscape) $)^{25-28}$. The iAtlas and the IO Landscape resource have been interlinked, enabling researchers to more readily understand the relationship between targeted proteins in IO therapy and the behavior of those targets in tumor tissue.

In IO Target Gene Expression Distributions, the distribution of gene expression values for the selected IO target, by sample group, is displayed in violin plots. Clicking on the expression distribution (violin plot) of a particular sample group, a histogram of the values is displayed.

The IO Target Annotations section provides a searchable table with IO targets and associated annotations. In the rightmost column, a link is provided to a view of the IO Landscape page, the selected target is highlighted in summary barcharts showing the number of agents and cancer types being studied for that target.

In the opposite direction, clicking on targets in the barcharts in the IO Landscape on CRI web pages brings up the target gene expression in iAtlas.

Tools

iAtlas Tools are accessible via the TOOLS tab on the iAtlas Portal. Modules in this space of the portal enable users to "bring their own data" for processing through immunogenomic algorithms that drive some of the results presented in the Analysis modules described above. 


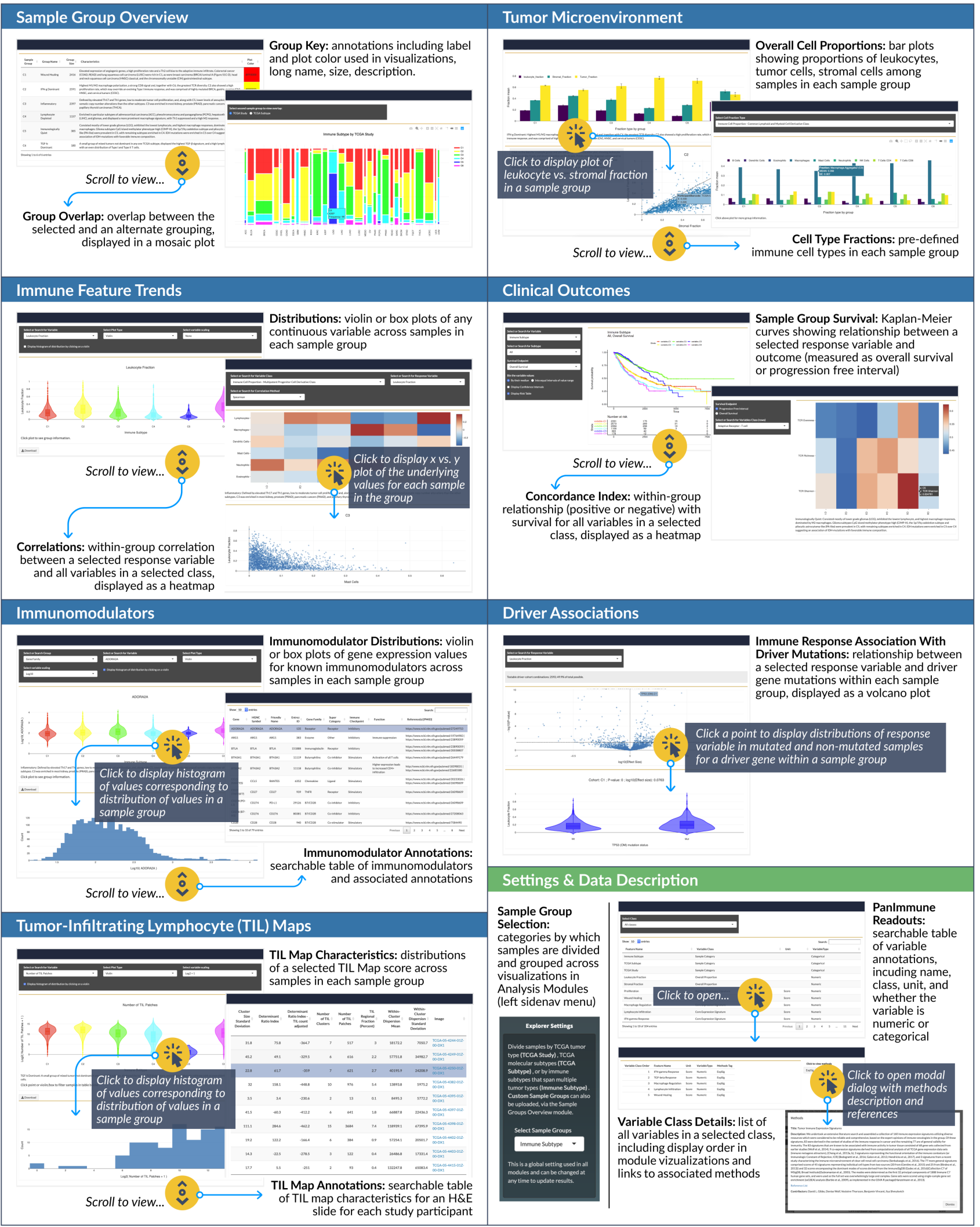

Figure 2. iAtlas Explorer. A range of Analysis modules (blue boxes above) are available that span from clinical to molecular and imaging data types. Within each module, interactive controls allow researchers to expand views, exposing underlying data and results. Settings are available (green box above) to select the sample groupings (TCGA Study, Disease Subtype, or Immune Subtype) which then propagate through modules. 
Immune Subtype Prediction: This tool performs classification of RNA-seq data into one of six immune subtypes as described in the Immune Landscape ${ }^{6}$ study. Using a new ensemble model based on $\mathrm{XGBoost}^{29}$, researchers can upload their own data for classification ${ }^{30}$. Each member of the ensemble was trained on a random subset of previously reported immune subtypes ${ }^{6}$ and features (described below) based on gene expression data from the TCGA PanCancer Atlas Initiative ${ }^{8}$. All code and methods have been confirmed as reproducible. An $\mathrm{R}$ package is available on GitHub (https://github.com/CRI-iAtlas/ImmuneSubtypeClassifier) ${ }^{30}$.

The submitted expression data-subsetted to the 485 genes that comprised the 5 signatures that produced the immune subtypes-are used to generate robust features of three types: quartiles, binary gene-pairs, and signature-pairs. For example, given a single sample, genes are binned into quartiles and given a bin label (quartile features). Then, similar to the "Top Scoring Pairs" classifier ${ }^{31}$, genes are paired, and given binary values depending on whether $\left(g_{i}>g_{j}\right)$ for two gene expression values, $g_{i}$ and $g_{j}$. Lastly, signature-pair features are calculated using the five immune subtype signatures, where $s_{m n}=\sum_{i j}\left(g_{i m}>g_{j n}\right) / k$, where $g_{i m}$ is gene $i$ from signature $m, g_{\text {jn }}$ is gene $j$ from signature $n$, and $k$ is the number of gene pairs considered resulting in a value between 0 and 1 . The features are computed independently for each sample, and do not require normalization across samples. These features are given to a trained XGBoost classifier which returns a probability of being in any of the six subtypes. Lastly, a "best call" is made with a final trained XGBoost classifier using the six probabilities as input. To validate the robustness of the classifier, TCGA data were processed using four different software pipelines and normalization, showing that classification performance was independent of the gene expression quantification method $^{30}$. Along with a downloadable table of results, visualizations are also provided. This tool is a convenient way for researchers to apply the methods of the Immune Landscape ${ }^{6}$ study to their own data without difficult statistical coding.

\section{Operation}

To use iAtlas, access the web app via https://www.cri-iatlas.org. The software can also be run locally on all platforms (Windows, Mac, Linux). To run the Shiny app locally, a working $\mathrm{R}$ installation with necessary libraries is required and an installation of RStudio is recommended.

To install and run the app locally:

1. Clone shiny-iatlas GitHub repository (git clone https://github.com/CRI-iAtlas/shiny-iatlas)

2. Open shiny-iatlas.Rproj in RStudio

3. Install packages. In the RStudio console, run:

$$
\text { renv: :restore() }
$$

4. Start the app by running:

$$
\text { shiny: : runApp () }
$$

\section{Use cases}

Reproducing published results and gaining information on underlying data

One of the initial motivations behind iAtlas was to provide an interactive platform that is able to reproduce figures published in the Immune Landscape ${ }^{6}$ manuscript but expands that with the ability to generate variations of those figures, for other choices of tumor samples and immune readouts of interest. As an example, in order to reproduce Figure $4 \mathrm{~A}$ from the Immune Landscape ${ }^{6}$ publication, which shows the correlation of DNA damage measures with the fraction of leukocytes in the tumor, we began by selecting the EXPLORE tab. We then opened the Immune Feature Trends module and selected the "Immune Subtype" option under Select Sample Groups in the Explorer Settings panel in the left menu. In the ensuing module page, at the Correlations section (Figure 3), we selected the "DNA Alterations" under Select or Search for Variable Class, "Leukocyte Fraction" under Select or Search for Response Variable, and the "Spearman" method under Select or Search for Correlation Method (each a separate dropdown menu). This produced a heatmap identical in content to Figure 4A in the Immune Landscape ${ }^{6}$ publication. However, the heatmap provides additional information on underlying data via interactivity: by clicking on a heatmap-cell, the underlying data is displayed in a scatterplot. Hovering a cursor over a point in the scatter plot reveals sample-level information.

Table 2 lists the particular manuscript figures (from the Immune Landscape $^{6}$ publication) that can be reproduced or adapted to specific research questions.

\section{Exploring new $\mathrm{IO}$ results}

With the iAtlas portal, scientists can explore and answer new questions based on specific research interests. For example, we asked: "What is the expression level of $P D-L 1$, a therapeutically important protein, in subtypes of breast cancer?" To answer this question, from the landing page, we first selected the "TCGA Subtype" sample group, followed by the "Breast Invasive Carcinoma (BRCA)" study subset. Next, we selected the Immunomodulators module (Figure 4). Based on a very quick scan of the drop down, we didn't see any names that matched our gene of interest, so we scrolled further down on the page to view the table of 'Immunomodulator Annotations'. By typing in the first few letters of a gene name (e.g., "PD...") into the 'Search' field, the table was filtered to a set of matching genes, and we could see that " $P D-L 1$ " is the Friendly Name for the gene "CD274" (the approved gene symbol on genenames.org). After returning to the Select or Search for Variable drop down menu above and selecting "CD274 (PD-L1)", we were able to see a display of violin plots showing the distributions of gene expression across BRCA molecular subtypes. We could then visually compare distributions between subtypes, noticing for example the elevated expression level in the Her2 subtype compared to Basal breast cancer. These comparisons can guide further characterization not only of how gene expression can differ between TCGA subtypes of breast cancer, but also how these subtype-specific differences might correlate with clinical outcomes, as investigated in other studies ${ }^{32-34}$. Using 


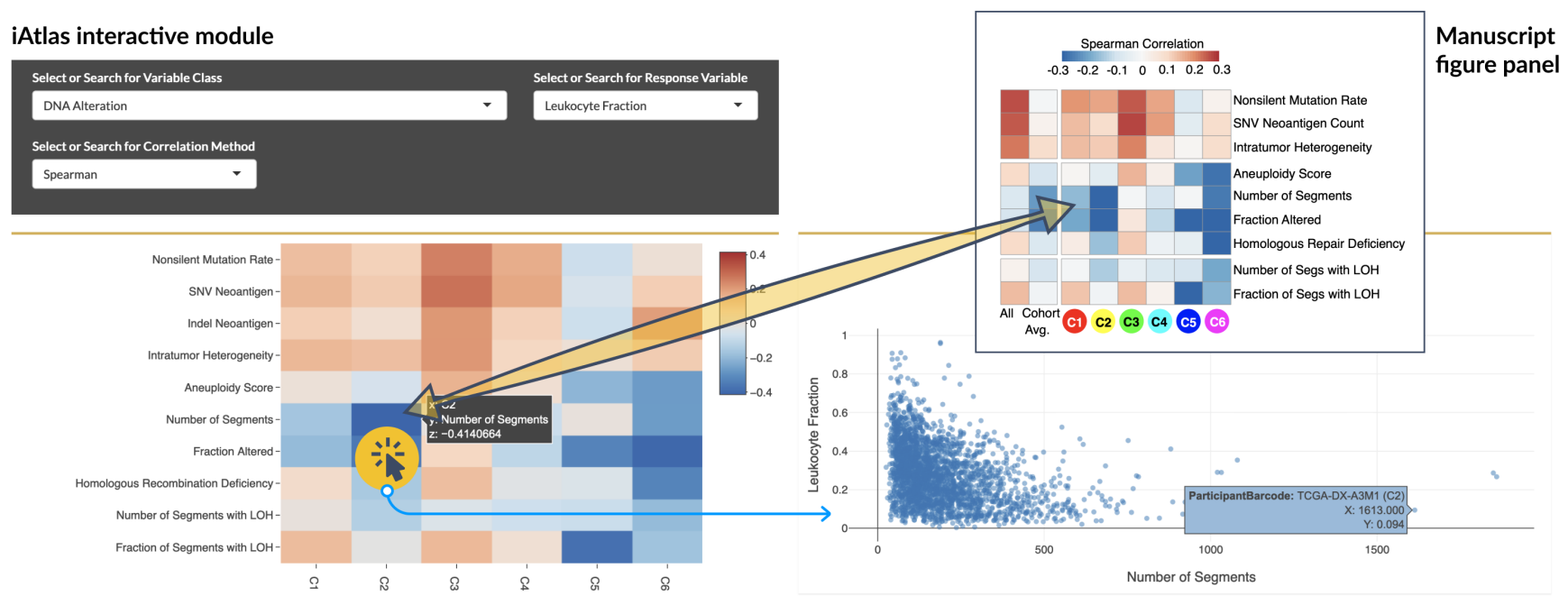

Figure 3. Visualization of the correlation of DNA damage in cancer with the degree of immune cell infiltration. Top right: Original manuscript figure panel from the Immune Landscape ${ }^{6}$ study. Bottom left: Equivalent figure generated in iAtlas. By selecting a specific heatmap cell (highlighted), the underlying data is displayed (Bottom right), using the selections shown. Individual points can be selected to get sample IDs and additional information (blue box).

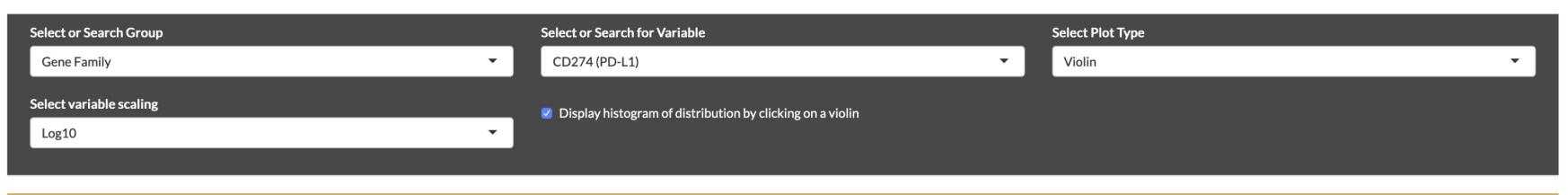

CD274 (PD-L1)

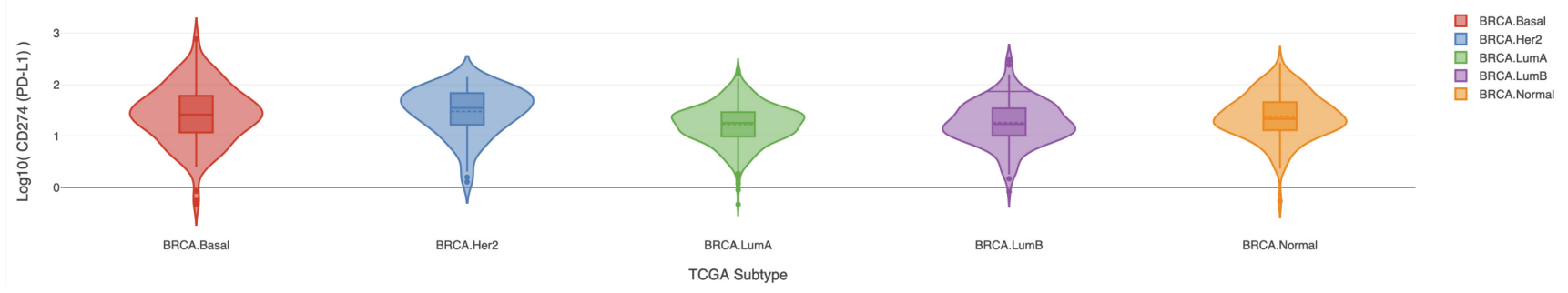

Figure 4. Visualization of the PD-L1 immunomodulator expression patterns in breast cancer subtypes. Selection for PD-L1 expression distributions displayed as violin plots within molecular subtypes of breast cancer, according to "PAM-50" classification. Elevated expression is seen in the HER2 subtype.

Table 2. Immune Landscape figures in iAtlas. iAtlas Analysis module (Column 1) and examples of figure panels (Column 2) in the Immune Landscape ${ }^{6}$ manuscript that can be generated using the module. Researchers can use this as a starting point for tailoring figures to their own interests.

\begin{tabular}{|l|l|}
\hline iAtlas Analysis module & Immune Landscape figures \\
\hline Sample Group Overview & 1D, S1B \\
\hline Tumor Microenvironment & 2A, 2C, S2A \\
\hline Immune Feature Trends & 1C, 2B, 4A \\
\hline Clinical Outcomes & 3A, 3B, 3C, S3A, S3B, S3C \\
\hline Immunomodulators & $6 \mathrm{~B}$ \\
\hline Driver Associations & 4D \\
\hline TIL Maps & 2D \\
\hline
\end{tabular}


this module and others, the researcher has the ability to answer new questions which could lead to developments in oncology research.

\section{Classification of immune subtypes on new data}

In order to classify any tumor-derived gene expression samples into immune subtypes ${ }^{6,30}$, users can select the TOOLS tab (top right), which leads to an interface containing notes, several links and the controls. In order to classify new data, we submitted data as a text file, in this case tab separated, with the first column containing gene IDs and later columns containing samples. A provided example file can be found in the description text. The first row of the data was a header containing sample IDs. Gene IDs can be either HGNC gene symbols (preferred), Entrez ID, or Ensembl identifiers. The locally available data was selected using the Browse button, and the file delimiter was selected, along with gene ID type, using drop down menus. Hitting the $G O$ button produced classifications, signature scores, and cluster probabilities, which were reported in a table that was downloaded as a csv, xlsx, or pdf file. In addition, a barplot with the frequency of predicted subtypes for the submitted data was displayed.

All data required to run the application and describe the Use Cases are available in GitHub and archived with Zenodo ${ }^{7}$.

\section{Conclusions}

CRI iAtlas is a platform that facilitates analysis and exploration of the tumor immune microenvironment by making IO-related data and tools accessible to the research community. iAtlas builds upon the comprehensive TCGA analysis of tumorimmune interactions on 10,000 tumors and illustrates how commonalities and differences of the immune response across 33 tumor types can provide clues for advancing therapeutics. iAtlas provides researchers with the tools to dive deeper into immunogenomic and clinical data and to develop and refine hypotheses regarding tumor-immune interactions that will empower researchers to gain insight and design the next generation of immuno-oncology treatment strategies.

\section{Data availability}

Source data

Original data files from the TCGA PanCancer Atlas publication can be found in the NCI Genomic Data Commons (https://gdc. cancer.gov/about-data/publications/panimmune) or the TCGA
PanCancer Atlas Data Mirror (https://isb-cancer-genomics-cloud. readthedocs.io/en/latest/sections/PanCancer-Atlas-Mirror.html.

\section{Extended data}

Zenodo: CRI iAtlas (Version 1.2.0). https://doi.org/10.5281/ zenodo. $3926757^{7}$.

Folder 'Data' contains all data required to run the application and describe Use Cases. This is also available on GitHub.

License: Apache License 2.0.

\section{Software availability}

Source code is available from GitHub: https:/github.com/ CRI-iAtlas/shiny-iatlas.

Source code for the specific version described at the time of publication: https://github.com/CRI-iAtlas/shiny-iatlas/releases/ tag/v1.2.0.

Archived source code at the time of publication: https://doi. org/10.5281/zenodo.39267577.

Hosted iAtlas application on shinyapps.io: https://isb-cgc.shinyapps.io/shiny-iatlas.

Pinned version of the hosted iAtlas app described at the time of publication: https://isb-cgc.shinyapps.io/iatlas_v1-2.

License: Apache License 2.0.

\section{Acknowledgements}

We are grateful to the Cancer Research Institute for supporting this work. We thank all collaborators in the TCGA PanCancer Atlas Immune Response Working Group, whose careful and thorough work generated the immune readouts displayed in iAtlas, and thank the NCI TCGA Program Office, Research Network, and PanCancer Atlas initiative for laying the foundation to this work. We thank Tai-Hsien Ou Yang, Eduard Porta-Pardo, Jun Tang, Vanessa Lucey, and Jill O'Donnell-Tormey, and the iAtlas user community for helpful suggestions and discussion on features and modules included in iAtlas. We also thank the TCGA participants who contributed samples used in this work.
1. Mellman I, Coukos G, Dranoff G: Cancer immunotherapy comes of age. Nature. 2011; 480(7378): 480-489.

PubMed Abstract | Publisher Full Text | Free Full Text

2. Fridman $\mathrm{WH}$, Pagès $\mathrm{F}$, Sautès-Fridman $\mathrm{C}$, et al:: The immune contexture in human tumours: impact on clinical outcome. Nat Rev Cancer. 2012; 12(4): 298-306.

PubMed Abstract | Publisher Full Text

3. Liu XS, Mardis ER: Applications of Immunogenomics to Cancer. Cell. 2017
168(4): 600-612.

PubMed Abstract | Publisher Full Text | Free Full Text

4. Binnewies $\mathrm{M}$, Roberts $\mathrm{EW}$, Kersten $\mathrm{K}$, et al:: Understanding the tumor immune microenvironment (TIME) for effective therapy. Nat Med. 2018; 24(5): 541-550. PubMed Abstract | Publisher Full Text | Free Full Text

5. Baruch EN, Berg AL, Besser MJ, et al.: Adoptive T cell therapy: An overview of obstacles and opportunities. Cancer. 2017; 123(S11): 2154-2162. PubMed Abstract | Publisher Full Text 
6. Thorsson V, Gibbs DL, Brown SD, et al:: The Immune Landscape of Cancer. Immunity. 2018; 48(4): 812-830.e14.

PubMed Abstract | Publisher Full Text | Free Full Text

7. Eddy J, Gibbs D, Lamb A, et al.: CRI iAtlas (Version 1.2.0). Zenodo. 2020. http://www.doi.org/10.5281/zenodo.3926758

8. Hutter C, Zenklusen JC: The Cancer Genome Atlas: Creating Lasting Value beyond Its Data. Cell. 2018; 173(2): 283-285. PubMed Abstract | Publisher Full Text

9. Chang W, Cheng J, Allaire JJ, et al:: shiny: Web Application Framework for R. $R$ package version 140. 2019. Reference Source

10. Cheng W: Modularizing Shiny app code. 2020. Reference Source

11. Wickham H, Averick M, Bryan J, et al:: Welcome to the Tidyverse. JOSS. 2019; 4(43): 1686 Publisher Full Text

12. Wickham H, François R, Henry L, et al:: dplyr: A Grammar of Data Manipulation. $R$ package version 083. 2019. Reference Source

13. Wickham H, Henry L: tidyr: Tidy Messy Data. R package version 100. 2019. Reference Source

14. Henry L, Wickham H: purrr: Functional Programming Tools. $R$ package version 033. 2019 Reference Source

15. Wickham H: stringr: Simple, Consistent Wrappers for Common String Operations. R package version 140. 2019 Reference Source

16. Müller K, Wickham H: tibble: Simple Data Frames. $R$ package version 213. 2019.

Reference Source

17. Mount J, Zumel N: wrapr: Wrap R Tools for Debugging and Parametric Programming. $R$ package version 192. 2019. Reference Source

18. Sievert C, Parmer C, Hocking T, et al:: plotly: Create Interactive Web Graphics via "plotly.js". R package version 490. 2019 Reference Source

19. Xie Y, Cheng J, Tan X: DT: A Wrapper of the JavaScript Library "DataTables". $R$ package version 09. 2019 Reference Source

20. Cheng J: crosstalk: Inter-Widget Interactivity for HTML Widgets. $R$ package version 100.2016. Reference Source

21. Chang W, Ribeiro BB: shinydashboard: Create Dashboards with "Shiny".
$R$ package version 071. 2018. Reference Source

22. Colaprico A, Silva TC, Olsen C, et al:: TCGAbiolinks: an R/Bioconductor package for integrative analysis of TCGA data. Nucleic Acids Res. 2016; 44(8): e71. PubMed Abstract | Publisher Full Text | Free Full Text

23. Liu J, Lichtenberg T, Hoadley KA, et al:: An Integrated TCGA Pan-Cancer Clinical Data Resource to Drive High-Quality Survival Outcome Analytics. Cell. 2018; 173(2): 400-416.e11.

PubMed Abstract | Publisher Full Text | Free Full Text

24. Saltz J, Gupta R, Hou L, et al.: Spatial Organization and Molecular Correlation of Tumor-Infiltrating Lymphocytes Using Deep Learning on Pathology Images. Cell Rep. 2018; 23(1): 181-193.e7.

PubMed Abstract | Publisher Full Text | Free Full Text

25. Tang J, Shalabi A, Hubbard-Lucey VM: Comprehensive analysis of the clinical immuno-oncology landscape. Ann Oncol. 2018; 29(1): 84-91. PubMed Abstract | Publisher Full Text

26. Tang J, Hubbard-Lucey VM, Pearce L, et al.: The global landscape of cancer cell therapy. Nat Rev Drug Discov. 2018; 17(7): 465-466. PubMed Abstract | Publisher Full Text

27. Tang J, Pearce L, O'Donnell-Tormey J, et al.: Trends in the global immunooncology landscape. Nat Rev Drug Discov. 2018; 17(11): 783-784. PubMed Abstract | Publisher Full Text

28. Yu JX, Hubbard-Lucey VM, Tang J: Immuno-oncology drug development goes global. Nat Rev Drug Discov. 2019; 18(12): 899-900.

PubMed Abstract | Publisher Full Text

29. Chen T, Guestrin C: XGBoost: A Scalable Tree Boosting System. In: Proceedings of the 22Nd ACM SIGKDD International Conference on Knowledge Discovery and Data Mining. KDD'16. New York, NY, USA: ACM. 2016; 785-794. Publisher Full Text

30. Gibbs DL: Robust classification of Immune Subtypes in Cancer. bioRxiv. 2020 Publisher Full Text

31. Geman D, d'Avignon C, Naiman DQ, et al.: Classifying gene expression profiles from pairwise mRNA comparisons. Stat Appl Genet Mol Biol. 2004; 3: Article19. PubMed Abstract | Publisher Full Text | Free Full Text

32. Jiang C, Cao S, Li N, et al.: PD-1 and PD-L1 correlated gene expression profiles and their association with clinical outcomes of breast cancer. Cancer Cell Int. 2019; 19: 233

PubMed Abstract | Publisher Full Text | Free Full Text

33. Padmanabhan R, Kheraldine HS, Meskin N, et al.: Crosstalk between HER2 and PD-1/PD-L1 in Breast Cancer: From Clinical Applications to Mathematical Models. Cancers (Basel). 2020; 12(3): 636. PubMed Abstract | Publisher Full Text | Free Full Text

34. Kurozumi S, Inoue K, Matsumoto $\mathrm{H}$, et al:: Clinicopathological values of PD-L1 expression in HER2-positive breast cancer. Sci Rep. 2019; 9(1): 16662. PubMed Abstract | Publisher Full Text | Free Full Text 


\title{
Open Peer Review
}

\section{Current Peer Review Status:}

\author{
Version 1
}

Reviewer Report 04 December 2020

https://doi.org/10.5256/f1000research.27745.r73635

(C) 2020 Reticker-Flynn N. This is an open access peer review report distributed under the terms of the Creative Commons Attribution License, which permits unrestricted use, distribution, and reproduction in any medium, provided the original work is properly cited.

\section{Nathan E. Reticker-Flynn}

Department of Pathology, Stanford University School of Medicine, Stanford, CA, USA

In their manuscript, Eddy et al. describe the interactive data portal iAtlas that accompanies the authors' 2018 publication in Immunity, "The Immune Landscape of Cancer". In that seminal paper, the authors present a compendium of analyses they performed to interrogate the diversity of immune states across human cancers using publicly available data from The Cancer Genome Atlas (TCGA). While the Immunity manuscript described the identification and characterization of six distinct "Immune Subtypes" and highlighted examples of key distinguishing features of the data sets, the accompanying iAtlas tool enables users to explore these rich analyses on their own, using parameters of their choosing. Comprising over 11,000 patients across 33 cancer types, these data represent an exceptionally comprehensive patient cohort from which a wide range of immunological insights can be gleaned.

iAtlas, which is available as both a web-based Shiny app and for offline implementation in R from GitHub, is a remarkably powerful and user-friendly portal through which researchers can easily interrogate the TCGA datasets for all of the immune analyses presented in their manuscript. The platform allows users to group their analyses by examining differences across TCGA cancer types, tumor subtypes (e.g. Luminal A vs. Her2 breast cancers), or their six defined Immune Subtypes. Furthermore, they have included the ability for users to define their own subsets and perform all of the included analyses across these subsets. The analyses within iAtlas include cellular readouts (e.g. degree of immune infiltration, fractions of particular leukocyte populations), molecular readouts (e.g. proliferation rates, gene expression, SNVs, CNAs, etc.), lymphocyte receptor (TCR/BCR) repertoires, network analyses, and tissue-level analyses (e.g. architectural characteristics). These variables can be investigated in the context of a wide range of parameters (e.g. clinical outcomes, correlations to driver gene mutations, etc.), designated as distinct modules, rendering the tool incredibly powerful with a near limitless number of potential questions to investigate in the field of tumor immunology. iAtlas is a remarkable tool with an extremely rich associated dataset whose elegant and straight-forward implementation render it accessible to researchers of all computational abilities.

I have no major concerns with this manuscript. What follows are a list of comments, minor 
concerns, and suggestions that should not preclude acceptance of the manuscript but rather serve as potential items to include in future releases of iAtlas:

1. In the Cell Type Fractions of the Tumor Microenvironment module, it would be helpful to include an option for the user to select which CIBERSORT immune types to include as a custom "Cell Fraction Type". For example, a user could select only memory resting CD4 T cells, memory activated CD4 T cells, naïve CD4 T cells, Tregs, and Tfh if they wanted to easily visualize the relative fractions of particular Th subsets within the CD4 compartment across groups. In some instances, it may be more biologically meaningful to compare these shifts within broad subsets without having to account for changes in unrelated populations (e.g. M2 macrophages).

2. In the Tumor Microenvironment module, when comparing leukocyte fraction to the total stromal fraction, there are typically a small number of samples where the leukocyte fraction exceeds the total stromal fraction, denoted as "estimation artifacts". What is the source of these artifacts? It may help to include a description of this in the legend.

3. As a general comment, in a variety of instances it might be informative if there would be a way to include statistics between groups, though I recognize that doing so could be computationally intensive and invalid in many instances. Nonetheless, given the large sample sizes I am often left wondering if modest differences between groups are statistically meaningful and where inferences can be drawn (e.g. an increase in Treg fraction from 0.024 in the "Inflammatory" subtype to 0.029 in the "TGF-b Dominant" subtype).

4. The Data Description tab might be aided by including a sentence or phrase within the table stating what each variable is without requiring following all of the methods links. For example, I am assuming the BCR/TCR Shannon incorporates both the richness and evenness to depict the diversity of the repertoire, but it might be helpful to state the meaning of the terms in the table.

5. For the Clinical Outcomes, it might be useful to include "Immune Subtypes" as a variable when analyzing TCGA Study so that users can evaluate the survival probabilities depending on immune subtype for a given cancer.

6. There appears to be a minor issue wherein no results can be displayed for the Concordance Index of the Clinical Outcomes analyses for most, if not all, variables when using TCGA Study for the Sample Groups.

7. The TIL Maps module is quite impressive and the ability to examine the histology from each of the patients along with the calculated metrics is an outstanding feature. Nonetheless, when one clicks one of the violin plots, the annotation lists seems only to display an individual patient rather than the entire list of patients for that group.

8. The Immune association with driver gene analysis is a powerful tool whose insights have far-reaching implications and represent an active area of research in tumor immunology. While the interactive volcano plot is highly informative and useful for identifying genes of interest, it would also be useful to be able to run the analysis in the opposite direction. If possible, it would be informative to be able to select a driver gene and see where it lies in the various volcano plots and which of the variables are correlated with the mutation. 
Is the rationale for developing the new software tool clearly explained?

Yes

Is the description of the software tool technically sound?

Yes

Are sufficient details of the code, methods and analysis (if applicable) provided to allow replication of the software development and its use by others?

Yes

Is sufficient information provided to allow interpretation of the expected output datasets and any results generated using the tool?

Yes

Are the conclusions about the tool and its performance adequately supported by the findings presented in the article?

Yes

Competing Interests: No competing interests were disclosed.

Reviewer Expertise: Tumor immunology, systems biology, immunotherapy, models of cancer, metastasis

I confirm that I have read this submission and believe that I have an appropriate level of expertise to confirm that it is of an acceptable scientific standard.

Reviewer Report 11 November 2020

https://doi.org/10.5256/f1000research.27745.r73636

(c) 2020 Goode D. This is an open access peer review report distributed under the terms of the Creative Commons Attribution License, which permits unrestricted use, distribution, and reproduction in any medium, provided the original work is properly cited.

David L. Goode

${ }^{1}$ Computational Cancer Biology Program, Peter MacCallum Cancer Centre, Melbourne, Vic, Australia

2 Sir Peter MacCallum Dept of Oncology, University of Melbourne, Melbourne, Vic, Australia

This article presents CRI iAtlas, a website with a suite of interactive tools for exploring and visualizing tumor immune microenvironment data collected by The Cancer Genome Atlas project.

It is based on robust, well-established $\mathrm{R}$ packages that are widely used for filtering, analysing and displaying large, multi-faceted data sets of the type used here. 
The design and implementation of the website are well described and the underlying code and data provided in readily accessible public repositories.

CRI iAtlas will provide a valuable resource for the cancer immunology field. The tools it provides will enable a range of users to quickly and easily access and visualize complex genomics and immunology data sets. The interface is user friendly and well documented, particularly for those without bioinformatics training.

The detailed instructions certainly made it easier to understand what each tool did the first time I tried to use them. However, they also kind of clutter the interface. It would be nice to be able minimize those panels without minimizing the entire module.

It would also be helpful to search the Driver Associations data for interactions involving certain genes of interest instead of having to scan through the volcano plots point by point.

But those are minor quibbles. Overall this is a slick and powerful online tool that will be welcomed by the field.

Is the rationale for developing the new software tool clearly explained?

Yes

Is the description of the software tool technically sound?

Yes

Are sufficient details of the code, methods and analysis (if applicable) provided to allow replication of the software development and its use by others?

Yes

Is sufficient information provided to allow interpretation of the expected output datasets and any results generated using the tool?

Yes

Are the conclusions about the tool and its performance adequately supported by the findings presented in the article?

Yes

Competing Interests: No competing interests were disclosed.

Reviewer Expertise: Cancer Genomics, Bioinformatics

I confirm that I have read this submission and believe that I have an appropriate level of expertise to confirm that it is of an acceptable scientific standard.

Reviewer Report 05 October 2020

https://doi.org/10.5256/f1000research.27745.r70105 
(c) 2020 Rouilly V. This is an open access peer review report distributed under the terms of the Creative Commons Attribution License, which permits unrestricted use, distribution, and reproduction in any medium, provided the original work is properly cited.

\section{Vincent Rouilly}

DATACTIX, Bordeaux, France

In this article, the authors introduce iAtlas, a web-based application that allows to browse a rich diversity of immune profiles, generated from the public TCGA dataset, and published in the landmark Immune Landscape study.

Further than simply giving the possibility to replicate the published figures, the application provides a great flexibility to explore the entire PanImmune feature matrix through sophisticated and interactive tools.

The modular architecture and the features of the software are clearly explained. Detailed instructions are provided. And, all the necessary information is given to run the application. Its source code repository on github is well structured. The documentation is very comprehensive, as it gives ample information on the underlying methods, as well as how to extend the software application.

It is a very valuable resource for the Immuno-oncology community.

Is the rationale for developing the new software tool clearly explained?

Yes

Is the description of the software tool technically sound?

Yes

Are sufficient details of the code, methods and analysis (if applicable) provided to allow replication of the software development and its use by others?

Yes

Is sufficient information provided to allow interpretation of the expected output datasets and any results generated using the tool?

Yes

Are the conclusions about the tool and its performance adequately supported by the findings presented in the article?

Yes

Competing Interests: No competing interests were disclosed.

Reviewer Expertise: Bioinformatics

I confirm that I have read this submission and believe that I have an appropriate level of expertise to confirm that it is of an acceptable scientific standard. 
The benefits of publishing with F1000Research:

- Your article is published within days, with no editorial bias

- You can publish traditional articles, null/negative results, case reports, data notes and more

- The peer review process is transparent and collaborative

- Your article is indexed in PubMed after passing peer review

- Dedicated customer support at every stage

For pre-submission enquiries, contact research@f1000.com 